\title{
La radio universitaria en el Ecuador
}

\section{The university radio in Ecuador}

*Lorena Katherine Álvarez Garzón ${ }^{1,}$ Alex Hernán Mullo López ${ }^{1}$, Melquiades Mendoza Pérez ${ }^{1}$

${ }^{1}$ Universidad Técnica de Cotopaxi, Facultad de Ciencias Humanas y de la Educación, Escuela de Comunicación Social. Av. Simón Rodríguez s/n Barrio El Ejido Sector San Felipe, provincia Cotopaxi. EC.05-01491.

Latacunga,Ecuador.*lorena.alvarez @utc.edu.ec; alex.mullo@utc.edu.ec; melquiades.mendoza@utc.edu.ec

Rec.: 17.02.2016. Acept.: 29.04.2016.

Publicado el 30 de diciembre de 2016

Rev. Cienc. Soc. y Econ. UTEQ (2016), 1(1): 93-106

\section{Resumen}

La investigación tiene como objetivo valorar el rol que cumplen las radios universitarias en el Ecuador a partir de la realidad dinámica en la construcción de otros mundos posibles a través de la vinculación entre la sociedad y la academia. La comunicación participativa e inclusiva busca acercar la ciencia a la comunidad, con la divulgación de investigaciones que incidan en el desarrollo y el buen vivir para lograr un empoderamiento tanto, científico como, cultural. En el proceso investigativo se evidencian las fortalezas y debilidades para alcanzar la meta, además del trabajo conjunto y solidario de las emisoras en la Red ecuatoriana que intercambia contendidos en un espacio de discusión y encuentro. La investigación se desarrolla a través de una evaluación cuali - cuantitativa en base al positivismo lógico mediante la cuantificación de datos. En referencia al contenido y estructura de la parrilla de programación se emplean el método hermenéutico y el registro narrativo a través de entrevistas no estructuradas a directivos de la red de radios universitarias del Ecuador, para comprender la realidad dinámica de las emisoras.

Palabras clave: comunicación, empoderamiento, cultura, programación, vinculación. 


\begin{abstract}
The Ecuadorian university seeks to guarantee the right to higher education through three axes: teaching, research and extramural activity to transform society. Various higher education institutions have seen on college radio, one of the means by which the university articulates with society and exerts its commitment to democratize knowledge and foster dissemination of research based on the need for local, regional development leading to the country's development. Various elements that propose a horizontal communication for development are analyzed, based on decolonial thought and communicology findings. The goal is to understand the dynamic reality of university radio
\end{abstract}

Keywords: college radio, empowerment, culture, programming, link.

\title{
Introducción
}

El presente trabajo pretende visibilizar la labor de las radios universitarias ecuatorianas, para ello toma como referencia la escuela crítica latinoamericana de la comunicación y la teología de la liberación que propone una comunicación para el desarrollo con igualdad de derechos y acceso al conocimiento, cuestionando y rechazando modelos dominantes de la información y estableciendo elementos decoloniales que José de Souza (citado por Torrico, 2011) propuso, el "pensamiento anormal" y la "desobediencia epistémica" para poder "ser nosotros mismos".

En relación a la comunicación y cultura, menciona que de los comunicadores dependerá que tengan éxito o fracasen los intentos de liberación cultural, como se enlaza con lo académico mientras la academia a través de su cuerpo docente, tiene un papel preponderante en está decolonización y su orientación hacia un desarrollo social, (Pasquali, 1990).

De modo, que existe una correlación entre el desarrollo nacional y la comunicación social, como lo detalla el sociólogo Daniel Lerner (citado por Beltrán, \& Ramiro, 2004) quien también habla de la misma interrelación que podría tener la universidad y el sentido de pertenencia con su conglomerado social, como constructora de ciencia - tecnología y la comunicación como inductora e indicadora del cambio social a través de sus diversos canales para llegar a las masas. 
Lo que ocurre a menudo bajo el nombre de comunicación es poco más que un monólogo dominante en beneficio del iniciador del proceso. La retroalimentación no se emplea para proporcionar la oportunidad de diálogo genuino. El receptor de los mensajes es pasivo y está sometido puesto que casi nunca se le brinda la oportunidad proporcional para actuar al mismo tiempo como verdadero y libre emisor; su papel esencial es el de escuchar y obedecer. Tan vertical, asimétrica y cuasi-autoritaria relación social constituye, una forma antidemocrática de comunicación... debemos... ser capaces de construir un nuevo concepto de la comunicación, un modelo humanizado, no elitista, democrático y no mercantilizado (Beltrán, 1978).

Este pensamiento de la comunicación se aplica a la difusión científica en y desde la academia, caracterizada por ser orientadora, que genera un cambio social y el buen vivir, reflexión crítica que plantea además la necesidad de construir otro tipo de procesos de comunicación que acerquen a la universidad y la comunidad mediante espacios que promuevan la investigación, la vinculación con la comunidad y el empoderamiento cultural y científico, (Arroyo, 2005).

En el Ecuador en los años 60 ya se presentaron iniciativas fuera de los estamentos universitarios como las escuelas radiofónicas populares del Ecuador ERPE en base al modelo de radio Sutatenza de Colombia, según (Yaguana, 2014, p. 39 ) con el objetivo principal de constituirse en una estación orientada "por y para la alfabetización "reivindicando por ejemplo a los pobladores de las zonas rurales y de sitios apartados de los núcleos urbanos", a partir de este y otros procesos las radios populares se agruparon alrededor de la CORAPE Coordinadora de Radios Populares del Ecuador con miras a una educación comunicacional desde y para la gente.

Luis Ramiro Beltrán (citado por Salmon, 2010) señala, acerca de la comunicación para el desarrollo, la aplicación planificada sistemática de instrumentos de comunicación social, para el logro de comportamientos de la población compatibles con sus necesidades de bienestar y con las finalidades de servicio expresadas en políticas, estrategias y planes de la sociedad pública, en este contexto las radios universitarias pueden convertirse en un interesante soporte para el empoderamiento de la ciencia y cultura.

La radio como medio de comunicación para la socialización de las ciencias y de aporte para el desarrollo social, ratifica su esencia educativa, es imposible pensar una radio que no promueva los valores identitarios del país y la dignidad humana. (San Félix, 1991, p. 22- 23) nos señala que la radio es uno de los modernos medios de difusión de ideas y de la divulgación de las ciencias, artes, etc. 


\section{La radio universitaria en el Ecuador}

En América Latina la radio universitaria nace en Argentina el 5 de abril de 1924, bajo el nombre de Radio Universidad Nacional de La Plata, al respecto Benito Nazar Anchorena (citado por Milito, C., \& Morzilli, 2015) menciona que:

A la Universidad de La Plata le corresponde la iniciativa de haber empleado una estación radiotelefónica no sólo como excelente elemento de enseñanza e investigación para la Radiotécnica sino también para fines de divulgación científica, o sea, como elemento de extensión universitaria. (Milito \& Morzilli, 2015, p. 4).

En Ecuador la radio universitaria ha estado ligada históricamente a las facultades de comunicación, una de las primeras fue la radio de la Universidad Central que nace aproximadamente en la década de los 70, "Luego de casi tres décadas de trámites y espera fue otorgada recientemente en el 2007, y que lamentablemente por cuestiones administrativas se la retiran en el 2013”. (Juan Manuel Aguiló. Entrevista personal N²1. Diciembre de 2015).

Para Romo (1989, p. 20) las radios universitarias son diferentes porque suenan de otro modo: los locutores son más respetuosos, muchas tienen como finalidad ser participativas, debido a que saben quién y cómo es su público.

Las radios universitarias en el país parten desde las conocidas radios base, que eran radios que se establecían en las universidades desde donde se promovía ciertos contenidos a nivel universitario (Juan Manuel Aguiló. Entrevista personal N². Diciembre de 2015).

La radio se presenta como una propuesta de vinculación con su entorno social y exposición del conocimiento y la cultura:

La problemática de la exclusión social, que popularizan la ciencia y el conocimiento que miman la música menos comercial e impulsan el trabajo de grupos emergentes, que aúnan cine, literatura, teatro y arte, que posibilitan el acceso y la participación de colectivos minoritarios, que fomentan los viajes culturales. (Perona; 2012, p43):

En 1975, 271 emisoras se constituyeron de manera legal, en tanto que para la época de los noventa según informes del Centro Internacional de Estudios Superiores de Comunicación para América Latina (CIESPAL), 342 emisoras universitarias se mantenían en funcionamiento. Para el 2015 la cifra ha aumentado notablemente; según datos presentados por el Consejo de 
Regulación de la información y Comunicación (CORDICOM) existen 564 radios privadas, 21 públicas, 32 comunitarias y 11 emisoras online.

La ley orgánica de Comunicación del año 2013, aprobada por la Asamblea Nacional de Ecuador establece la redistribución de las frecuencias radiofónicas y las divide en un 33 por ciento para medios privados, 33 por ciento para medios públicos y 34 por ciento para medios comunitarios. Si se busca llegar a plasmar esta meta, los datos obtenidos en 2015 con el 92 por ciento que corresponden a radios privadas, el 5 por ciento para públicas y el 3 por ciento para comunitarias es decir aún no se logra cumplir la normativa.

A lo señalado se suma que aún no se instituye una normativa reglamentaria que permita la inclusión de las radios universitarias en el espectro radioeléctrico, a decir de docentes entrevistados a cargo de las emisoras, no hay claridad si una radio universitaria deber ser pública, comunitaria por pertenecer a una comunidad universitaria, o privada según la naturaleza de cada Institución de educación superior.

Se considera importante un trabajo conjunto para reivindicar el compromiso social e ineludible que tienen las universidades de convertirse en un espacio académico y comunitario para estrechar vínculos con la sociedad mediante la comunicación radiofónica y buscar mecanismos idóneos en el ámbito jurídico y administrativo que facilite la obtención de frecuencias abiertas que permitan un mayor alcance.

\section{El trabajo en Red}

Otros autores destacan el trabajo en red como parte de la ingeniería en comunicación social en América Latina, Galindo Cáceres, (2012). El 12 de noviembre de 2009, en la ciudad de San José, Costa Rica, se constituyó la Red de Radios Universitarias de Latinoamérica y el Caribe (RRULAC). Es un ente conformado por redes nacionales de instituciones de educación superior de América Latina, con fines de desarrollo académico y de comunicación, sin fines de lucro.

Esta organización está conformada actualmente por: 9 países, 5 redes nacionales, 5 radios asociadas, 155 universidades, 211 radiodifusoras universitarias. De hecho Annuies (citado por Ruiz, 2005) menciona que:

La decisión de conformar un sistema y no otro tipo de organización fue tomada por las propias instituciones participantes con miras a poder establecer acuerdos interinstitucionales que posibilitaran la capacitación y actualización del personal 
dedicado, al desarrollo de la radio, así como también para facilitar el intercambio de materiales la elaboración conjunta de estudios y documentos con el fin de mejorar tanto la difusión y promoción de la cultura como el conocimiento que se genera al interior de las propias instituciones educativas (ANNUIES, 2002).

Ecuador, forma parte de la RRULAC desde el tres de abril del 2014 por medio de la Red de Radios Universitarias del Ecuador (RRUE) entidad formada el 29 de octubre del 2003, en la ciudad de Quito, con el firme propósito de convertirse en un espacio académico, comunitario, creado para estrechar vínculos entre las instituciones educativas universitarias del país que actúen en el campo de la comunicación radiofónica.

Las radios universitarias son un medio de comunicación y difusión desde las IES en el Ecuador que pretenden aportar de manera significativa en la formación de los futuros comunicadores y comunicadoras a la vez que buscan convertirse en medios de divulgación que condicionen el empoderamiento científico y cultural, (Galindo, 2012).

La Universidad debe salir de su claustro para utilizar estrategias nuevas que permitan una comunicación horizontal por medio de las radios universitarias, comunicación entendida según (Villanueva \& Beltrán, 2009, p. 105) como “...el proceso de interacción social democrática que se basa sobre el intercambio de símbolos por los cuales los seres humanos comparten voluntariamente sus experiencias bajo condiciones de acceso libre e igualitario, diálogo y participación" ya que generalmente difunden la actividad y los resultados de sus investigaciones por medio de publicaciones científicas, de acceso limitado hacia la población en general, por ende es necesario generalizar la ciencia, la tecnología a través de la oralidad que menciona (Cebrian, 2001, p. 32).

En el contacto humano mediante los sonidos para ahuyentar soledades, para acompañar, para informar para entretener, y en suma, para seguir manteniendo al ser humano vinculado al elemento primigenio y de mayor riqueza a lo largo de su historia como es la tradición mediante la comunicación oral. Y el medio idóneo para el efecto es la radio por su poder de penetración, por su economía, por su accesibilidad a toda la población con instrucción académica o no (Dede, 2000).

La radio universitaria entonces busca utilizar la palabra como eje de la comunicación oral, acompañada de la música, fiel inspiración creativa humana, los efectos y el silencio, elementos fundamentales para el espacio armónico de sonidos, de vivencias, de construcción de 
identidades culturales, de reflexiones y propuestas de soluciones, de conocimiento y arte, así es la radio, un fiel reflejo de la cultura de los pueblos, (Pérez, 2004).

La radio universitaria busca la comunicología de la liberación que mencionan (Villanueva \& Beltrán, 2009, p. 105)...la comunicación no debe ser una herramienta para la irreverente manipulación de los seres humanos con el afán de satisfacer los intereses creados de unos pocos. Tampoco debe la comunicación emplearse para preservar una injusta estructura social; debe usársela para transformarla de manera que prevalezcan la justicia y la paz.

De esta manera, Pasquali (1990, p. 28) reconfirma que en el futuro habrá que ser más realistas, más pragmáticos, más convincentes, concretos, exigentes. Tenaces y eficientes. Reconfirmemos solemnemente nuestro propósito de no cesar hasta que nuestras comunicaciones les lleguen la hora de la Democracia, de la Utilidad Social y de la Calidad.

Las radios universitarias, mediante una comunicación horizontal participativa y colaborativa, pueden generar procesos para lograr el empoderamiento de la ciencia, de tal manera que estén al alcance de todos y todas, propender al cambio social, cultural y económico mediante procesos planificados de divulgación de la cultura y el saber científico. Pérez Rosas (citado por Pena \& Narváez, 2014) señala que de esta manera, estas emisiones radiales significan nuevas oportunidades y medios didácticos para suscitar en la población una mejor comprensión acerca de las diversas situaciones del entorno"

Desde la Universidad, se requiere aprovechar las nuevas plataformas tecnológicas y la posibilidad del trabajo colaborativo multidisciplinar y diverso que logra realizarse mediante el trabajo articulado, por ello nace la red de radios universitarias del Ecuador una propuesta de organización conjunta.

A continuación presentamos un estudio realizado sobre las radios universitarias en el Ecuador y la función que cumplen para la divulgación científica y cultural, mediante un análisis de parrilla de programación y contenidos, audiencia y la participación de Docentes y estudiantes en la elaboración de productos comunicacionales.

\section{Metodología}

La investigación se desarrolla a través de una evaluación cuali - cuantitativa en base al positivismo lógico mediante la cuantificación de datos en referencia al contenido y estructura de la parrilla de programación y la metodología cualitativa naturalista y el pensamiento 
hermenéutico con el registro narrativo a través de entrevistas no estructuradas a directivos de la red de radios universitarias del Ecuador, para comprender la realidad dinámica de las emisoras.

El análisis de tipo cuantitativo se realiza a través de encuestas aplicadas a 17 directores o representantes de las radios universitarias, en donde se busca establecer al talento humano que participa en las radios, el tipo de contenido que se produce, estrategias de interacción con la audiencia y su formato de transmisión. En tanto que el análisis cualitativo se obtiene con la entrevista no estructurada y la comprensión interpretativa de los directivos de la Red de Radios Universitarias del Ecuador, para obtener sus reflexiones sobre la importancia de la radio universitaria y su rol dentro de la sociedad.

\section{Resultados y discusión}

Para la realización del estudio se toman cuatro indicadores en los cuales se obtienen los siguientes resultados:

\section{Rol de la radio universitaria}

De la investigación se desprende que las radios tienen un afán de servicio por su naturaleza educativa y de transferencia de conocimiento, para los académicos y estudiantes este medio se constituye en una nueva estrategia para divulgar los conocimientos y experimentos que se desarrollan en el aula en base a las necesidades del entorno social.

Los programas de divulgación científica y cultural son parte esencial de los contenidos en las parrillas de programación, se presentan los resultados de las investigaciones que se ponen al alcance de la audiencia a la vez que se cumple con el rol de vinculación con el sector social y productivo.

Las radios se constituyen además en un espacio valido para la realización de prácticas sistémicas y pre profesionales de los estudiantes de las facultades y carreras de comunicación social tradicionalmente ligadas a este tipo de emisoras.

En la investigación se visibilizó el trabajo en red de las 17 emisoras universitarias, se cumple con el objetivo central de la Red de Radio de Ecuador de contribuir al acercamiento, desarrollo y fortalecimiento de las relaciones académicas y comunicacionales entre todos los integrantes. 
Pese a las iniciativas de las radios universitarias a nivel del país aún no se logran proyectos cooperativos que potencien su impacto con el aporte de producciones interactivas y de contenido educativo transmitidas en todas las radios que forman parte de la red, es decir no se llega al empoderamiento cultural de los contenidos, los estudios evidencian que aún no se llega a influir para una verdadera participación e interacción social, lo que se explica en el siguiente indicador.

\section{Sobre la producción y participación}

El estudio arroja que el 75 por ciento de universidades que poseen radios ofertan la carrera de comunicación social o periodismo, por ende su programación está ligada a las practicas que realizan los estudiantes de la carrera con la supervisión de su docente, ambos se transforman en los principales protagonistas de la parrilla de programación, llama la atención que en cifras se demuestra que quienes salen al aire en igual porcentaje son docentes (46\%) y estudiantes (46\%), y solo un 8 por ciento profesionales externos.

Respecto a los radioescuchas o interlocutores, los medios radiales universitarios cuentan con una "audiencia cautiva" conformada por la comunidad universitaria, su mayor impacto es intra - institucional con poco impacto en el resto de la sociedad. Se evidencia que las radios universitarias si bien es cierto poseen contenidos tendientes a la formación y educación, la divulgación del conocimiento y la cultura, estos aún no establecen estrategias y mecanismos o nuevas tecnologías para poder llegar a tener una audiencia participativa desde la sociedad en general, pese a que el 52 por ciento de encuestados considera a la sociedad como la principal audiencia de la radio.

En la dinámica de la radio, si existe un proceso de comunicación horizontal entre docentes, estudiantes y personal administrativo de la misma institución, logra la confluencia de diversos pensamientos y transformarse en un medio de vinculación, aunque es un esfuerzo aún insuficiente para lograr una verdadera comunicación participativa con los actores sociales como protagonistas.

Del registro narrativo a través de entrevistas no estructuradas a directivos de la red de radios universitarias del Ecuador se desprende, que algunas radios llegaron únicamente a difundir un contenido que solo servía a unas elites del conocimiento, sin un tratamiento que permita pasar del lenguaje académico a un lenguaje popular que comunique y divulgue a las audiencias de 
las radio universitarias, existe una iniciativa para trabajar en productos comunicacionales de fácil entendimiento para la difusión de contenidos que o pierdan la esencia de la universidad.

\section{Estructura de la programación}

En relación a las parrillas de programación, en su mayoría están conformadas por programas radiales tipo mosaico que aborda temas de salud, ciencia y tecnología, cultura, identidad, inclusión social, informativos universitarios entre otros. Este tipo de programas tienen una duración promedio de una hora semanal o diaria. Las radio revistas o ómnibus son escasos debido al esfuerzo en pre producción y producción que se debe realizar y a la falta de recursos humanos y económicos.

Generalmente la parrilla de programación evidencia la identidad y los objetivos del medio de comunicación universitario que en este estudio reflejan el carácter de servicio y vinculación con la sociedad a través del empoderamiento del conocimiento y la cultura.

\section{Contenidos de la programación}

En referencia a la producción de la radio, el 83 por ciento tiene una finalidad formativa y supervisada por docentes y personal de la universidad, y un 17 por ciento corresponde a una actividad de ocio que responde a los intereses de determinados grupos de alumnos o profesores.

En cuanto a los contenidos que más se emiten en la radio universitaria se pueden considerar que los temas de ciencia, tecnología, salud, calidad de vida, interculturalidad y pluriculturalidad sobresalen del resto de programas, generalmente docentes investigadores y especialistas en las temáticas son los actores de estos espacios, la parte técnica y de conducción es apoyada por los docentes y estudiantes de las diversas facultades de comunicación, los cuales crean mecanismo como el lenguaje sencillo y claro característico de los medios radiofónicos, en este tipo de medios se combinan géneros como el periodístico y musical en cuanto a los formatos radiofónicos se evidencia el uso de las entrevistas, tertulias, noticias, debates, en microprogramas de 60 minutos.

En base a los objetivos de la radio, la mayoría de emisoras universitarias utilizan la música como elemento de entretenimiento y captación del público, el concepto de una radio universitaria con música clásica esta fuera de la órbita ecuatoriana. Hecho inusual ya que en el paradigma establece que este tipo de medios emiten música no comercial. Por su parte, el pop, la balada y la música ecuatoriana predominan en la parrilla de programación 


\section{Formato de accesibilidad si se le pone nuevos medios y nuevas narrativas}

Un elemento importante para la radio universitaria ha sido la llegada del internet y la oportunidad de un dial sin fronteras e infinito, las múltiples dificultades para conseguir la adjudicación de una frecuencia impulsaron a las instituciones de educación superior a apuntar sus esfuerzos en formatos como el streaming y el podcast para la transmisión vía on line.

El acceso a la red digital permite a las universidades generar a través de las nuevas tecnologías medios universitarios sin restricciones o engorrosos trámites legales que obstaculicen su transmisión, el estudio demuestra que en el Ecuador el 71 por ciento de radios universitarias emiten su señal vía internet y apenas un 29 por ciento por frecuencia abierta.

Ya que se habla de nuevas tecnologías, es necesario reflexionar sobre las diversas posibilidades de retroalimentación y participación que estas nos brindan.

El Internet, permite la anexión del oyente al discurso radiofónico, a instaurar programaciones en bases a sus necesidades, por ende la audiencia tiene una participación preponderante en los contenidos debido a la multiplicidad de herramientas como el chat, el correo electrónico, las redes sociales, y el podcast que en forma particular permiten al oyente tener a la mano la información o producto sin necesidad de estar pendiente de la hora de la transmisión del programa, elemento que ha ayudado a multiplicar las posibilidades de distribución del conocimiento y la cultura. En las radios universitarias de Ecuador apenas el 47 por ciento de las estaciones aprovechan estos elementos.

\section{Discusión de los resultados}

El trabajo colaborativo y la capacidad de generar producciones en conjunto, aún no se ha explotado al máximo, deberá asumir con mayor fuerza la Red de Radios del Ecuador para contribuir al acercamiento, desarrollo y fortalecimiento de las relaciones académicas y comunicacionales entre todos los integrantes de la red.

En el Ecuador existe un esfuerzo desde las Universidades para crear medios de comunicación como la Radio universitaria y realizar un trabajo articulado por medio de una red que promueve la difusión de la ciencia y cultura al alcance de todos y todas, transformándose en un nexo entre la academia y la comunidad.

Se entiende que la radio universitaria es un instrumento válido para la formación de los futuros comunicadores mediante un proceso organizado de prácticas, pero tiene una doble función pues 
acerca a la gente a los adelantos científicos y tecnológicos, la Ciencia no se queda en las aulas sino que se difunde hacia la sociedad para promover su desarrollo.

Es imperante buscar mecanismos urgentes que permitan facilitar los procesos para que se pueda contar con licencias que permitan acceder a mayor cantidad de población, de tal manera que se pueda incidir de manera positiva pensando en un desarrollo basado en el buen vivir y el la participación.

Las experiencias de radios Universitarias señalan como la comunicación puede aportar de manera significativa para darle nuevos significados y sentidos de la ciencia y generar procesos de transformación social y equidad.

E ecuador tienen una gran riqueza de cultura oral, las radios universitarias pueden contribuir a que la sabiduría popular no se pierda, apoyar desde la comunicación para la construcción y fortalecimiento de la identidad, y a la par generar procesos de educación no formal para mejorar la calidad de vida de las personas, lo que puede ser extensivo al continente americano.

\section{Conclusiones}

La producción radial desde la academia aporta nuevos medios pedagógicos para la comprensión y difusión de temáticas que coadyuven la solución de problemáticas sociales, a la propagación de la ciencia y la cultura de los pueblos.

Las radios universitarias a nivel del país deben reforzar su función social, desarrollar proyectos cooperativos que potencien su impacto, priorizar la divulgación y promover los valores culturales del país, se suma a esto también el apoyar los vínculos que se generan desde las redes de radio universitaria para unirse a ellas y aportar con producciones interactivas y de gran contenido educativo.

La radio universitaria ecuatoriana necesita de un cambio orientado a lograr un empoderamiento de la ciencia y la cultura en la comunidad y sociedad.

\section{Referencias bibliográficas}

Arroyo, CM. (2005). Escuela Latinoamericana de Comunicación y el Pensamiento Crítico de Antonio Pasquali. Revista Alaic, Año II, N², enero-junio, pp. 22-30 
Beltrán, LR., \& Ramiro, L. (2004). La comunicación y el desarrollo democráticos en Latinoamérica. Comunicación, 126, 79-94.

Beltrán, S., \& Ramiro, L. (1978, October). Communication between the USA and Latin America: a case of cultural domination. In World Media Conference, 1st, New York (pp. 19-22).

Cebrián, HM. (2001). La radio en la convergencia multimedia, Gedisa, España, 2001.

Dede, C. (comp.) (2000), Aprendiendo con tecnología, Barcelona, Paidós.

Galindo, LJ. (2012). Comunicología e ingeniería en comunicación social del conflicto y la articulación. Apuntes para un programa de trabajo en comunicología de la comunicación estratégica Intersticios Sociales, núm. 3, marzo-agosto, 2012, pp. 1-35, El Colegio de Jalisco, Zapopan, México

Galindo, LJ. (2011). "Methodology in Social Communication Engineering. General Notes on the Methodological Program:Diagnosis, Design of Social Intervention, and Technical Implementaton of the Intervention". Francisco Vargas Serrano, Antonina Ivanova Boncheva, Gerrit Meijer, Benjamin Burgos Flores (eds.). New Challenges, New Methodologies. Proceedings of the xi,ISINI Conference.México: Pearson Educación de México, Universidad de Sonora.

Lozano, C. \& Martín Pena, D. (2014). Investigaciones sobre radio universitaria: Presente, pasado y futuro. EDMETIC, Revista de Educación Mediática y TIC, 3(1), 8-25.

Milito, C. \& Casajús, L. (2011) Las radios universitarias argentinas en Internet: relevamiento, desarrollos, modelos y enfoques. Question, 1(30). Recuperado

Molina, JL. (2001), El análisis de redes sociales: una introducción, Barcelona, Bellaterra

Narváez, CE., \& Pena, DM. (2012). Las radios universitarias, más allá de la radio: las TIC como recursos de interacción radiofónica (Vol. 230). Editorial UOC.

Novelli, C., \& Hernando, Á. (2011). Las radios universitarias. Trayectoria histórica y panorama mundial. J. Ignacio Aguaded y Paloma Contreras (Coords.), La radio universitaria como servicio público para una ciudadanía democrática. A Coruña: Netbiblo, 12-24.Milito, C., \& Morzilli, E. La programación de las radios universitarias argentinas.

Pasquali, A. (1990) NOMIC: de la academia a la acción. En: Documentos del Encuentro "EL NOMIC" Cara al Año 200. Lima, Perú, 26- 28 de noviembre de 1990. Lima, IPAL

Pena, DM., \& Narvaez, CE. (2014). Contenido, estructura y función social en la programación de las radios universitarias. Edmetic, 3(1), 26-43. 
Perona, JJ. (2012). Las emisoras universitarias en el contexto digital: programación, nuevos medios y hábitos de escucha. (pp. 37-52). En C. Espino y D. Martín (coords.). Las radios universitarias, más allá de la radio. Las TIC como recursos de interacción radiofónica. Barcelona: UOC.

Pérez, RLD. (2004). La radiodifusión universitaria y los circuitos cerrados de radio en las instituciones de educación superior: una propuesta para la expresión de los estudiantes. Revista Mexicana de Ciencias Políticas y Sociales, 47(192), 153-186.

Ruiz, MR. (2005). Radios y redes: la radio en las instituciones de educación superior. Reencuentro, 44(29).

Romo-Gil, C. (1989). Las radiodifusoras no comerciales en México. Una diversidad de opciones en crecimiento. Revista Renglones, 15, 19-23.

San Félix, A. (1991). La radiodifusión en la mitad del mundo: Apuntes históricos. Ecuador. Editorial Nacional.

Salmón, LRB. (2010). Comunicación para la salud del pueblo: una revisión de conceptos básicos. Estudios sobre las culturas contemporáneas, (31), 17-65.

Torrico, E. (2011). Emancipar a Comunicação para sustentar a paz-DOI: http://dx. doi. org/10.15603/2175-7755/cs. v32n54p191-210. Comunicação \& Sociedade, 32(54), 173-190.

Vázquez, M. (2010). Jóvenes y Radio Universitaria: un cercamiento a las estrategias formativas de dos países. Resultados de la encuesta La participación juvenil en radiodifusoras universitarias de México y España'. II Congreso Internacional de la Asociación Española de Investigadores de la Comunicación (AE-IC) Málaga 2010.

Villanueva, ET. (2009). Luis Ramiro Beltrán: Por una" Comunicología de liberación”. Chasqui. Revista Latinoamericana de Comunicación, (105).

Yaguana, H. \& Delgado, W. 2011. 85 años de la radiodifusión en Ecuador. Editorial Quipus. Ecuador. 